\title{
A vitamina D em pacientes críticos: uma revisão da literatura
}

\section{The vitamin $D$ in critically ill patients: a literature review}

\author{
Valdevino Pedro Messias Netoํ. Lucian Batista de Oliveira ${ }^{1}$. José Olivandro Duarte de Oliveira ${ }^{1}$. \\ 1 Universidade Federal de Campina Grande (UFCG), Campina Grande, Paraíba, Brasil.
}

\section{RESUMO}

A vitamina D é lipossolúvel e tem suas ações influenciadas por numerosos moduladores, sendo indispensável para saúde esquelética, neurológica, imunológica e cardiovascular, havendo cada vez mais apontamentos de que a hipovitaminose $\mathrm{D}$ aumenta o risco de morbimortalidade hospitalar. Este trabalho objetivou realizar uma revisão da literatura recente, com estudos publicados entre 2009 e 2018 em importantes bancos de dados de saúde, sobre a deficiência da vitamina D em pacientes críticos e as suas possíveis consequências. Utilizou-se os descritores "cuidados críticos" and "vitamina D". Foram selecionados 8 trabalhos. Constatou-se maiores índices da deficiência nutricional nos pacientes em terapia intensiva do que em pacientes hígidos, em diferentes faixas etárias, havendo sugestão de que a hipovitaminose estaria associada a maiores taxas de mortalidade. A suplementação da vitamina D nessas condições ainda não foi amplamente estudada, mas observa-se uma tendência a ser benéfica, não trazendo eventos adversos. Conclui-se que a hipovitaminose D provavelmente está associada a piores desfechos clínicos em pacientes graves, mostrando a necessidade de grandes estudos multicêntricos randomizados para comprovação da necessidade da sua investigação e da sua correção.

Palavras-chave: Vitamina D. Cuidados críticos. Deficiências nutricionais.

\section{ABSTRACT}

The vitamin D is liposoluble and have its actions influenced by numerous modulators, being indispensable for skeletal, neurological, immunological and cardiovascular health, with increasing attention to the fact that hypovitaminosis D increases the risk of hospital morbidity and mortality. This study aimed to review the recent literature, with studies published between 2009 and 2018 in important health databases, about vitamin D deficiency in critically ill patients and their possible consequences. The descriptors "critical care" and "vitamin D" were used. Eight articles were selected. There were higher rates of nutritional deficiency in intensive care patients than in healthy patients in different age groups, suggesting that hypovitaminosis would be associated with higher mortality rates. Vitamin D supplementation in these conditions has not yet been extensively studied, but there is a tendency to be beneficial, not bringing about adverse events. It is concluded that hypovitaminosis D is probably associated with worse clinical outcomes in critically ill patients, showing the need for large randomized multicenter studies to prove the need for its investigation and its correction.

Keywords: Vitamin D. Critical care. Deficiency diseases.

Autor correspondente: Lucian Batista de Oliveira, Hospital Universitário Alcides Carneiro, Rua Carlos Chagas, sem número, São José, Campina Grande, Paraíba, Brasil. CEP: 58429-900. Telefone: +55 83 2101-5575. E-mail: lucianbaptist@gmail.com

Conflito de interesses: Não há qualquer conflito de interesses por parte de qualquer um dos autores.

Recebido em: 25 Set 2018; Revisado em: 25 Out 2018; Aceito em: 05 Nov 2018. 


\section{INTRODUÇÃO}

O estado nutricional do paciente crítico tem influência direta na evolução e desfecho clínicos, sendo um dos grandes desafios na unidade de terapia intensiva (UTI) e incorporando-se como parte do cuidado integral ao enfermo. ${ }^{1,2}$

Carências nutricionais têm íntima relação com o processo inflamatório do paciente grave, de forma que a doença crítica se associa a desarranjos metabólicos que culminam no processamento anormal de nutrientes, tornando benéfico um maior aporte de alguns oligoelementos. ${ }^{1,3-5}$ Atualmente, vem se destacando nesse cenário a vitamina $\mathrm{D}$, alvo de constantes estudos. ${ }^{6,7}$

A vitamina D é lipossolúvel e tem características de um hormônio, com similaridade estrutural com os esteroides. Suas formas principais são o colecalciferol (vitamina D3) e o ergocalciferol (vitamina D2), de origens animal e vegetal, respectivamente. Ambas são convertidas em 25-hidroxivitamina-D (25OHD) no fígado e posteriormente em 1,25-di-hidroxivitamina D $\left[1,25(\mathrm{OH})_{2} \mathrm{D}\right]$ nos rins, sendo esta última a forma biologicamente ativa, também chamada de calcitriol. A vitamina D3, além de obtida pela dieta, pode ser sintetizada na pele a partir de exposição suficiente à radiação solar ultravioleta B. ${ }^{6,8,9}$

Os níveis de vitamina D no organismo são influenciados por numerosos moduladores, como paratormônio, cálcio, fósforo e fator de crescimento de fibroblastos-23. Em condições normais, tais níveis são avaliados pela $250 \mathrm{HD}$ sérica, cuja concentração é 500 a 1000 vezes maior que a de $1,25(\mathrm{OH})_{2} \mathrm{D}$, porém, diretamente proporcional. ${ }^{7,9}$

A vitamina D age através de vias genômicas e não genômicas pleiotrópicas, sendo indispensável para saúde esquelética, desenvolvimento neurológico, sistema imunológico e aparelho cardiovascular. A manifestação clássica da sua deficiência é o raquitismo em crianças e a osteomalácia em adultos, havendo novos apontamentos de que essa hipovitaminose também aumente o risco de doenças cardiovasculares, pulmonares, neoplásicas e de morbimortalidade hospitalar. ${ }^{6-9}$

Diante do crescente interesse pela otimização da terapia adjuvante do paciente crítico por meio do suporte nutricional e das promissoras observações a favor da vitamina D nesse contexto, observa-se a relevância de estudos que abordem especificamente este tema, justificando a necessidade de novas pesquisas e um balanço dos atuais achados literários sobre o assunto.

O presente trabalho tem como objetivo realizar uma revisão narrativa da literatura recente sobre a deficiência de tal micronutriente no paciente crítico e os seus possíveis efeitos na evolução e prognóstico dos doentes.

\section{MÉTODO}

A revisão da literatura se caracteriza enquanto estudo que analisa a produção bibliográfica em determinada área temática, dentro de um recorte de tempo, fornecendo uma visão geral ou um relatório do estado da arte sobre um tópico específico, evidenciando novas ideias, métodos e subtemas que têm recebido maior ou menor ênfase na literatura selecionada. ${ }^{10}$

Para a obtenção dos artigos usados neste estudo, foram utilizados os seguintes critérios de refinamento: estudos publicados entre 2009 e 2018; escritos em português, inglês ou espanhol; exclusão de textos coincidentes e seleção dos textos com total alusão à temática.

Foram então acessados os bancos de dados do Periódico CAPES, Scientific Electronic Library Online (SciELO), OneFile (GALE), Scopus (Elsevier), Science Citation Index Expanded (Web of Science), MEDLINE/PubMed (NLM) e ScienceDirect Journals (Elsevier). Utilizou-se os descritores "cuidados críticos" and "vitamina D", os quais estão contidos na lista dos descritores em Ciências da Saúde (DeCS), além dos seus correspondentes nas línguas inglesa e espanhola, estando eles contidos nos campos título, resumo ou assunto.

O tratamento dos dados foi realizado por meio da análise qualitativa dos artigos selecionados, inicialmente por dois diferentes autores em sistema duplo-cego, havendo, nos casos de discordância, uma nova análise por parte de um terceiro autor.

Algumas fragilidades encontradas para a coleta de dados foram a não disponibilidade de textos completos em parcela significativa dos trabalhos analisados e também o fato de alguns deles não conterem informações sobre as abordagens metodológicas e objetivos das reflexões, sendo preciso uma leitura na íntegra dos artigos e demais produções.

Somando-se todas as bases de dados, de acordo com os critérios e a metodologia supracitada, foram selecionados 8 artigos, após a exclusão de textos repetidos. Construiu-se então a revisão com a leitura na íntegra e a análise crítica de todos os artigos que foram selecionados.

\section{RESULTADOS E DISCUSSÃO}

\section{Hipovitaminose D e mortalidade na terapia intensiva}

Dos trabalhos selecionados, 4 trataram diretamente da hipovitaminose $\mathrm{D}$ como preditora de mortalidade em pacientes críticos da faixa etária adulta, em situações em que não houve administração da vitamina nos grupos estudados. ${ }^{11-14}$

Estudo de coorte realizado em UTI de hospital de ensino em Roma, na Itália, analisou todos os pacientes internados com sepse grave ou choque séptico que tiveram os seus níveis de $25 \mathrm{OHD}$ dosados nas primeiras 24 horas após admissão. Considerou-se como ponto de corte para deficiência de vitamina $\mathrm{D}$ valores séricos de $20 \mathrm{ng} / \mathrm{ml}$, e níveis $<7 \mathrm{ng} / \mathrm{ml}$ como extremamente baixos. Dos 127 pacientes analisados, 
93,5\% apresentavam $25 \mathrm{OHD}<20 \mathrm{ng} / \mathrm{ml}$ e $53,3 \%$ valores $<7 \mathrm{ng} / \mathrm{ml}$. Os pacientes com níveis extremamente baixos apresentaram uma taxa de mortalidade relacionada à sepse significativamente maior do que o restante da amostra (50,9\% versus $26 \%$ ), além de uma duração mais longa de ventilação mecânica e de necessidade de suporte vasopressor, o que apontou que a hipovitaminose $\mathrm{D}$ grave pode ser um forte preditor de mortalidade relacionada à sepse. ${ }^{11}$

Por sua vez, outro estudo italiano, realizado em Florença, comparou pacientes admitidos em UTI por duas diferentes causas: sepse grave/choque séptico (92 pacientes) e vítimas de trauma (72 pacientes). Ambos os grupos tiveram baixos níveis de vitamina $\mathrm{D}$ à admissão, com média de $10,1 \mathrm{ng} / \mathrm{ml}$ no grupo de pacientes sépticos e de 18,4 ng/ml em pacientes traumatizados, havendo diferença estatisticamente significativa entre os níveis de 25OHD de tais grupos. Porém, por meio de regressão logística multivariada, não se observou a hipovitaminose D como fator significativamente correlacionado a uma maior taxa de mortalidade em pacientes sépticos, sendo então sugerido novos estudos para esclarecer o papel prognóstico desse micronutriente na sepse. ${ }^{12}$

Outro estudo, observacional e prospectivo, que também considerou valores $<20 \mathrm{ng} / \mathrm{ml}$ para caracterização de deficiência de vitamina $\mathrm{D}$, constatou que $82,3 \%$ de uma amostra de 130 pacientes graves, com necessidade de ventilação mecânica, apresentavam baixos níveis de 25OHD, e que o tempo médio de sobrevida foi significativamente mais longo naqueles com níveis suficientes da vitamina: 24,2 dias $( \pm 16,5)$, contra 15,3 dias $( \pm 12,4)$ no grupo com hipovitaminose. Diante disso, indicou-se que a vitamina $\mathrm{D}$ pode ser um biomarcador ou cofator de sobrevivência em pacientes gravemente enfermos. ${ }^{13}$

Mais um estudo observacional, sendo este com uma maior amostra e realizado em 2 centros americanos, demonstrou que a deficiência de vitamina $\mathrm{D}$ no início do tratamento intensivo foi preditiva de mortalidade a curto e a longo prazo. Utilizou-se valores diferentes dos estudos anteriormente abordados para caracterizar a deficiência de vitamina D: níveis de $25 \mathrm{OHD} \leq 15 \mathrm{ng} / \mathrm{ml}$ categorizaram a deficiência de vitamina $\mathrm{D}$, considerando-se insuficiência quando valores de 16 a $29 \mathrm{ng} / \mathrm{ml}$ e suficiência quando $\geq 30 \mathrm{ng} / \mathrm{ml}$. Dos 1325 pacientes contidos na amostra, 50,4\% eram deficientes de vitamina D, observando-se nesta parcela uma maior mortalidade em 30 dias, assim como em 90 e em 365 dias após o início do tratamento crítico. ${ }^{14}$

Em síntese, nota-se uma tendência à hipovitaminose $\mathrm{D}$ em pacientes criticamente doentes, de forma que três dos quatro estudos supracitados ${ }^{11,13,14}$ mostram associação entre maiores taxas de mortalidade e/ou menor tempo de sobrevida com níveis inadequados da vitamina, principalmente quando esses valores se apresentam extremamente baixos. O outro trabalho não encontrou associação entre hipovitaminose $\mathrm{D}$ e mortalidade específica por sepse, ${ }^{12}$ porém, comparou dois grupos de pacientes graves (quadro séptico e vítimas de trauma), inclusive observando médias baixas da vitamina em ambos, não contrapondo a sugestão de que a deficiência do micronutriente seja um preditor de mortalidade e um determinante no desfecho clínico de pacientes críticos como um todo.

\section{Deficiência de vitamina D em pacientes graves nos extremos de idade}

A abordagem específica da hipovitaminose D nos pacientes críticos nos extremos etários também foi realizada. ${ }^{15,16}$

Uma produção abordou esse déficit nutricional na faixa etária pediátrica. Trata-se de um estudo observacional e prospectivo que comparou níveis de 25OHD em 156 crianças nas primeiras 12 horas de internação em UTI com os de 289 crianças saudáveis. A deficiência de vitamina D foi definida como valores de $25 \mathrm{OHD}<20 \mathrm{ng} / \mathrm{ml}$. As crianças internadas em UTI foram ainda comparadas em relação aos escores de mortalidade pediatric risk of mortality III (PRISM III) e pediatric index of mortality 2 (PIM 2), sendo divididas em 2 grupos de acordo com os percentis: $>$ p75 em pelo menos um dos escores ( 33 crianças) e $<$ p75 em ambos (123 crianças). A prevalência de hipovitaminose $\mathrm{D}$ em crianças internadas foi de $29,5 \%$, enquanto em crianças saudáveis foi de $15,6 \%$. A razão de chance para hipovitaminose $\mathrm{D}$ em crianças internadas em UTI foi de 2,26. Não se observou, no entanto, associação entre a deficiência da vitamina e maior previsão de escores de risco de mortalidade, tempo de internação e suporte inotrópico ou respiratório nas crianças criticamente doentes. ${ }^{15}$

Em relação à faixa etária geriátrica, um estudo francês avaliou a associação entre o tempo de internação em unidade de tratamento agudo geriátrico e os níveis de 250HD na admissão em uma amostra de 253 pacientes, com uma média de idade de 86,2 anos. Considerou-se hipovitaminose D valores de $25 \mathrm{OHD} \leq 50 \mathrm{nmol} / \mathrm{L}(20 \mathrm{ng} / \mathrm{ml})$. Observou-se que os idosos com déficit desse oligoelemento apresentaram um tempo de internação em média 3 dias maior que aqueles com níveis adequados. Adiciona-se ainda o achado de associação linear entre a concentração sérica de vitamina $\mathrm{D}$ e o tempo de internação, sugerindo que qualquer aumento nos níveis da vitamina associa-se a uma diminuição no período de hospitalização, independente dos valores iniciais. ${ }^{16}$

Nos extremos de idade, portanto, vê-se, assim como em adultos jovens e de meia idade, uma possível propensão à hipovitaminose $\mathrm{D}$ em pacientes gravemente enfermos. Apesar de não ter sido constatado em crianças uma associação desse déficit nutricional com tempo de internação ou com outros indicadores de morbimortalidade, na população idosa verificou-se um menor período de hospitalização, reforçando ainda mais a sugestão de que os níveis da vitamina podem ser um relevante fator na evolução clínica e a importância da realização de estudos intervencionistas, com diferentes faixas de idade.

\section{A suplementação de vitamina D no paciente crítico}

Dois dos trabalhos selecionados abordaram a administração da vitamina D nos pacientes submetidos a cuidados intensivos. ${ }^{17,18}$ 
$\mathrm{O}$ primeiro deles avaliou a resposta à administração de $25 \mathrm{OHD}$ e $1,25(\mathrm{OH})_{2} \mathrm{D}$ em pacientes gravemente enfermos, comparando-os com indivíduos saudáveis (doadores de sangue). Observou-se níveis séricos de vitamina $\mathrm{D}$, tanto das formas hidroxiladas quanto das não hidroxiladas, significativamente menores nos doentes críticos. O grupo de pacientes graves foi ainda subdividido em grupo controle (12 indivíduos), submetidos a altas doses de vitamina D via oral $(\mathrm{n}=11)$ e sujeitos à administração via endovenosa de vitamina $\mathrm{D}(\mathrm{n}=11)$. Constatou-se que os pacientes gravemente doentes possuem grande deficiência de vitamina $\mathrm{D}$, a qual foi facilmente corrigida pela administração oral de 25OHD. A administração endovenosa de 1,25(OH) 2 também demonstrou promover um aumento nos níveis séricos da vitamina, sugerindo que a deficiência funcional do metabolismo do micronutriente nos enfermos graves provavelmente possa também ser corrigida pela administração dessa forma ativa da vitamina. ${ }^{17}$

O mais recente deles, de 2017, trata-se de uma revisão sistemática com metanálise, que envolveu estudos randomizados, englobando 716 pacientes. Observou-se uma mortalidade significativamente menor nos pacientes que fizeram uso da vitamina D comparando-se com o grupo para o qual se administrou placebo: $32 \%$ versus $40 \%$. Além disso, não foram encontradas diferenças nos eventos adversos entre os 2 grupos. ${ }^{18}$

\section{REFERÊNCIAS}

1. Guimarães HP, Assunção MS, Carvalho FB, Japiassú AM, Veras KN, Nácul FE, et al. Manual de medicina intensiva AMIB. São Paulo: Atheneu; 2014.

2. Fontoura CS, Cruz DO, Londero LG, Vieira RM. Avaliação nutricional de paciente crítico. Rev Bras Ter Intensiva. 2006 [Acesso em: 21 Maio 2018];18(3):298-306.

3. Manzanares W, Dhaliwal R, Jiang X, Murch L, Heyland DK. Antioxidant micronutrients in the critically ill: a systematic review and meta-analysis. Crit Care. 2012;16(2):R66.

4. Jensen GL, Wheeler D. A new approach to defining and diagnosing malnutrition in adult critical illness. Curr Opin Crit Care. 2012;18:206-11.

5. Marino PL. Compêndio de UTI. 4. ed. Porto Alegre: Artmed; 2015.

6. Amrein K, Oudemans-van HM, Berger MM. Vitamin therapy in critically ill patients: focus on thiamine, vitamin C, and vitamin D. Intensive Care Med. 2018;44(11):1940-4.

7. De Pascale G, Antonelli M, Quraishi SA. Vitamin D, hospitalacquired infections and mortality in critically Ill patients: emerging evidence. In: Vincent JL, editor. Annual update in intensive care and emergency medicine 2017. [place unknown]: Springer International Publishing; 2017. p. 169-83.

8. Harvey RA, Ferrier DR. Bioquímica Ilustrada. 5. ed. Porto Alegre: Artmed; 2012.

9. Borba VZ, M Mañas NC, Moreira CA. Deficiência de
Dessa forma, essa suplementação vitamínica ganha notoriedade em âmbito de cuidados intensivos, tendo em vista as numerosas sugestões de que pacientes críticos apresentam baixos níveis de 25OHD e de que a sua reposição pode diminuir a morbimortalidade, sendo crucial o estabelecimento de novas evidências ao seu favor para uma possível implementação na prática clínica.

\section{CONCLUSÕES}

Diante do apresentado, observa-se a existência de relevantes apontamentos sobre o favorável papel da vitamina $\mathrm{D}$ em pacientes graves, havendo notória tendência a níveis séricos baixos da vitamina nesses enfermos e uma possível relação dessa deficiência com maiores taxas de morbidade e mortalidade nas mais diversas faixas etárias.

A carência de grandes estudos multicêntricos e randomizados sobre o tema limitam as evidências e o uso devidamente embasado da suplementação de vitamina D neste contexto, abrindo margem para a realização de novos ensaios com tais moldes, diante dos animadores achados benéficos naqueles com níveis adequados de $25 \mathrm{OHD}$ em estudos observacionais e da não constatação de eventos adversos expressivos referentes à administração da vitamina naqueles com a hipovitaminose dentro dos poucos estudos que a utilizaram, no contexto de cuidados críticos. Enxerga-se, portanto, uma potencial nova arma no manejo de pacientes sob cuidados intensivos.

vitamina D: por que, quando e como tratar? In: Vilar L, editor. Endocrinologia clínica. 6. ed. Rio de Janeiro: Guanabara Koogan; 2016. p. 1029-36.

10. Souza MT, Silva MD, Carvalho R. Integrative review: what is it? How to do it? Einstein (São Paulo). 2010;8(1):102-6.

11. De Pascale G, Vallecoccia MS, Schiattarella A, Di Gravio V, Cutuli SL, Bello G, et al. Clinical and microbiological outcome in septic patients with extremely low 25-hydroxyvitamin D levels at initiation of critical care. Clin Microbiol Infect. 2016;22(5):456e7-13.

12. Cecchi A, Bonizzoli M, Douar S, Mangini M, Paladini S, Gazzini $\mathrm{B}$, et al. Vitamin D deficiency in septic patients at ICU admission is not a mortality predictor. Minerva Anestesiol. 2011;77(12);1184-9.

13. Arnson Y, Gringauz I, Itzhaky D, Amital H. Vitamin D deficiency is associated with poor outcomes and increased mortality in severely ill patients. QJM. 2012;105(7):633-9.

14. Braun AB, Gibbons FK, Litonjua AA, Giovannucci E, Christopher $\mathrm{KB}$. Low serum 25-hydroxyvitamin D at critical care initiation is associated with increased mortality. Crit Care Med. 2012;40(1):6372 .

15. Rey C, Sánchez-Arango D, López-Herce J, Martínez-Camblor P, García-Hernández I, Prieto B, et al. Vitamin D deficiency at pediatric intensive care admission. J. Pediatr (Rio J). 2014;90(2):135-42.

16. Hélard L, Mateus-Hamdan L, Beauchet O, Annweiler C. Hypovitaminosis D in geriatric acute care unit: a biomarker of longer length of stay. Dis Markers. 2013;35(5):525-9. 
17. Mata-Granados JM, Vargas-Vasserot J, Ferreiro-Vera C, de Castro MD, Pavón RG, Gomez JM. Evaluation of vitamin D endocrine system (VDES) status and response to treatment of patients in intensive care units (ICUs) using an on-line SPE-LC-MS/ MS method. J Steroid Biochem Mol Biol. 2010;121(1-2):452-5.
18. Putzu A, Belletti A, Cassina T, Clivio S, Monti G, Zangrillo A, et al. Vitamin D and outcomes in adult critically ill patients. A systematic review and meta-analysis of randomized trials. J Crit Care. 2017;38:109-14.

\section{Como citar:}

Messias VP Neto, Oliveira LB, Oliveira JO. A vitamina D em pacientes críticos: uma revisão da literatura. Rev Med UFC. 2019 jul-set;59(3):48-52. 